\section{Practical Dosimetry Considerations for Small MLC-Shaped Electron Fields at 60 cm SSD}

\author{
Déte Van Eeden*๑, Karl N. Sachse², Freek C.P. Du Plessis ${ }^{1}$
}

\begin{abstract}
Superficial tumours can be treated with megavoltage electron beams. The underlying tissue can be spared through the steep dose fall-off gradients over a range of a few centimetres.

An accurate Monte Carlo model for an Elekta Precise was determined and dose distribution was simulated. Dosimetric parameters were calculated to set guidelines for tumour irradiation.

Elekta Precise multi-leaf collimators (MLC), which shaped electron fields were investigated using a benchmarked Monte Carlo model. BEAMnrc modelled the Elekta Precise and results were benchmarked against measurements. Percentage depth dose and beam profile data were simulated within $2 \% / 2 \mathrm{~mm}$ accuracy of the measured data. The DOSXYZnrc code simulated the 3-D dose data in water between 4 and $15 \mathrm{MeV}$. The relative $\left(\mathrm{P}_{80-20}\right)$ penumbra, percentage depth dose (PDD), range to $90 \%$ of dose maximum $\left(\mathrm{R}_{90}\right)$, dose fall-off range $\mathrm{R}_{80-20}(\mathrm{DFR})$, and the percentage bremsstrahlung dose (BSD), were extracted from the simulated data.

The relative penumbra ranged from $90 \%$ to $10 \%$ at $6 \mathrm{MeV}$ and $15 \mathrm{MeV}$, respectively. $\mathrm{R}_{90}$ values ranged between $0.8 \mathrm{~cm}$ at $4 \mathrm{MeV}$ and $4.5 \mathrm{~cm}$ at $15 \mathrm{MeV}$. The DFR ranged between $0.8 \mathrm{~cm}$ at $4 \mathrm{MeV}$ and $3.5 \mathrm{~cm}$ at $15 \mathrm{MeV}$. The BSD was the highest for low beam energies and small fields.

Developed guidelines indicated that intermediate-sized MLC fields are most suited for therapy since they have lower BSD, longer $\mathrm{R}_{90}$, shorter DFR but larger $\mathrm{P}_{80-20}$. The DFR increases and $\mathrm{R}_{90}$ decreases for small fields at higher beam energies and more distal tissue will receive doses $>20 \%$.
\end{abstract}

Citation: Van Eeden D, Sachse KN, Du Plessis FCP. Practical Dosimetry Considerations for Small MLC-Shaped Electron Fields at $60 \mathrm{~cm}$ SSD. $J$ Biomed Phys Eng. 2022;12(1):101-108. doi: 10.31661/jbpe.v0i0.2004-1097.

\section{Keywords}

Electrons; Multi-Leaf Collimator; Energy; Monte Carlo Method; Radiometry

\section{Introduction}

uperficial tumours can be treated with megavoltage electron beams

S due to sparing of the underlying tissues caused by the steep dose fall-off gradients. In some surface treatments, electron beams irradiate less surrounding tissue when compared to oblique photon beams e.g. during photon treatment of the parotid and some breast tumours where there is the significant normal tissue involvement in tangent field beam arrangements.

Electron therapy uses energy and intensity modulated electron beam radiotherapy (MERT) [1, 2] for treatment of parotid, paraspinal, and
${ }^{1} \mathrm{PhD}$, Department of Medical Physics, Faculty of Health Sciences, University of the Free State, Bloemfontein, South Africa

${ }^{2} \mathrm{MSc}$, Department of Medical Physics, Faculty of Health Sciences, University of the Free State, Bloemfontein, South Africa
*Corresponding author: Déte Van Eeden

Department of Medical Physics, Faculty of Health Sciences, University of the Free State

P.O. Box 339, Bloemfontein, 9300 South Africa E-mail: vaneeden.d@ufs. ac.za

Received: 7 April 2020 Accepted: 2 June 2020 
breast tumours [3, 4]. Electron beam energy selection is based on the depth range to $90 \%$ dose maximum $\left(\mathrm{R}_{90}\right)$, covering the tumour bed which deliver a therapeutic dose [5] $R_{90}$ increases for increasing field size and reaches a saturation value for fields that are larger than $10 \times 10 \mathrm{~cm}^{2}$ [6]. Monte Carlo (MC) MERT simulation studies were also done to investigate photon multi-leaf collimator (MLC) or electron MLC beam delivery [7, 8]. One study investigated dose delivery through a photon MLC at $60 \mathrm{~cm}$ source-to-surface distance (SSD) for different carcinoma treatments [9]. EGSnrc and macro Monte Carlo (MMC) simulations have been done for a Synergy S, Clinac 23EX and TrueBeam linear accelerators.

Another type of electron beam therapy is segmented-field electron conformal therapy (ECT). It involves the delivery of electron beams through a number of abutted fields [10].

Applicators or MLCs can be used to collimate the electron beam. The question now arises: If single MLCs collimated electron fields are used for small lesion treatments, what are the dosimetric consideration (guidelines) and how does it depend on the field size and beam energy?

The dosimetric consideration includes the electron range from the surface to $90 \%$ (dose fall-off $\left(\mathrm{R}_{90}\right)$, the lateral distance between the $80 \%$ and $20 \%$ isodose lines (penumbra), the depth range from $80 \%$ to $20 \%$ dose fall-off, defined here as the dose fall-off range (DFR), and percentage bremsstrahlung dose (BSD) since MLCs which collimated electrons are delivered at $60-70 \mathrm{~cm}$ SSD [11].

The aims of this study was as follows: I) To develop an accurate Monte Carlo model for an Elekta Precise that is benchmarked against measurements with Gafchromic film and water tank data II) To extract dosimetric parameters from the 3-D dose distributions to develop guidelines for using single electron fields for small lesion irradiation.

\section{Material and Methods}

Treatment head and water tank simulation

The EGSnrc-based BEAMnrc and DOSXYZnrc MC codes were used in this study [12, 13]. BEAMnrc was used to model an Elekta Precise treatment head. Electron beam fields were formed by the MLC and jaws, with sizes defined at $60 \mathrm{~cm}$ SSD. The MLC is 7.5 $\mathrm{CM}$ thick and has rounded leaf tip edges. The treatment head has a pair of MLC backup jaws ( $3 \mathrm{~cm}$ thick) and a pair of jaws $(7.5 \mathrm{~cm}$ thick), all composed of a heavy metal alloy.

MC transport setting includes energy cutoffs of 0.010 and $0.521 \mathrm{MeV}$ for photons and electrons, respectively. Photon forcing, range rejection, bremsstrahlung splitting, Rayleigh scattering and bound Compton scattering were not requested. Atomic relaxations and spin effects were implemented. The electron-step algorithm and boundary cross algorithm (BCA) were set as PRESTA-II and EXACT.

The electron beam sources were circular with a Gaussian fluence distribution. The source energy spectra were adjusted to match measured electron beam dose distributions at $95 \mathrm{~cm}$ source-collimator-distance (SCD). Here, the electron applicator was included in the treatment head and phase space files were scored at $95 \mathrm{~cm}$ SCD and used in DOSXYZnre simulation. The same MC transport setting was used as described above. The simulated water tank dimensions were $30 \times 30 \times 30 \mathrm{~cm}^{3}$ with voxel sizes of $0.1 \times 0.1 \times 0.2 \mathrm{~cm}^{3}$ in the $\mathrm{x}, \mathrm{y}$, and $\mathrm{z}$ directions.

Beam profile- and percentage depth dose (PDD) data were extracted from the simulated 3D dose in the water tank and compared with measurements. The matching criterion was set at $2 \% / 2 \mathrm{~mm}$.

MLC collimated electron beam simulation

Simulations were performed for field sizes 
MLC Segment Selection in Segmented-Field ECT

between $1 \times 1 \mathrm{~cm}^{2}$ and $20 \times 20 \mathrm{~cm}^{2}$.

Dose parameters were calculated from $3 \mathrm{D}$ dose data from DOSXYZnrc. The water tank surface was set at SSD $=60 \mathrm{~cm}$. PDD data were extracted from which $\mathrm{R}_{90}$, DFR and BSD parameters were obtained. $\mathrm{P}_{80-20}$ values were obtained from beam profiles at an effective depth of $0.1 \mathrm{~cm}$.

\section{Film measurement of MLC collimated electron beams}

MLC fields were set up and EBT2 film (Lot\#03181402) was used to obtain dose profiles for 4, 6, 8, 10 and $15 \mathrm{MeV}$ for a range of fields at $\mathrm{SSD}=60 \mathrm{~cm}$ using 100 monitor units (MUs) in a RW3 solid water phantom. It was placed at $0.1 \mathrm{~cm}$ and $0.9 \mathrm{~cm}$ below the surface for 4,6 and $8 \mathrm{MeV}$ data. For 10 and $15 \mathrm{MeV}$ data, the second measurement depth was 1.9 $\mathrm{cm}$. After $24 \mathrm{~h}$, the films were scanned on an EPSON perfection V330 document scanner in TIFF image format and 48 bit-depth and resolution of 50 dpi.

The data of red channel TIFF image were converted into dose using a method described by O'Reilly and co-workers [14].

The resulting dose profile images were analysed using ImageJ r1.46 (National Institute of Health). Corresponding DOSXYZnrc dose profile data were compared with the benchmark film data.

\section{Dosimetric Guidelines for small MLC field selection}

Field size for small lesion irradiation is important to ensure adequate tumour dose coverage and sparing distal organs at risk (OARs). Guidelines were developed for radiation planning of small lesions. The DFR is an important guideline since it describes the dose falloff between $\mathrm{R}_{80}$ and $\mathrm{R}_{20}$, indicating distal dose penetration into potentially OAR tissue close to the tumour bed.

The BSD is the bremsstrahlung dose present in the PDD curve and indicates the dose that distal normal (OAR) tissue receives for the field size under consideration. A criterion of less than $5 \%$ is set as a guideline.

The $R_{90}$ to DFR ratio is a scale, indicating the therapeutic dose range over the dose penetration range. It is a useful parameter to consider where OARs are close to the tumour bed and a different field size should rather be considered to avoid excessive dose into the OAR (at the expense of more lateral normal tissue dose).

The penumbra is defined as the lateral distance between the $80 \%$ and $20 \%$ isodose lines, and its magnitude depends on the depth at which it is taken.

\section{Results}

MC and measured dose comparison for applicator collimated electron beams

The MC simulation of the Elekta Precise accelerator produced depth dose data that compared within $2 \% / 2 \mathrm{~mm}$ from measure depth dose data as seen in Figure 1.

MC and film dose comparison for MLC collimated electron beams

Comparison between film and MC dose profiles are shown in Figure 2. The variance was within $1 \%$ and $2.3 \%$ for the $\mathrm{MC}$ data and film data, respectively and also too small to be shown on the graphs. The correspondence between the film and MC data was within $2 \%$ $/ 2 \mathrm{~mm}$.

$\mathrm{P}_{80-20}$ and $\mathrm{R}_{90}$

$\mathrm{P}_{80-20}$ for MLC collimated electron beams decreases due to the decrease in the field size. $\mathrm{P}_{80-20}$ was measured at $0.2 \mathrm{~cm}$ depth and ranged between $1.2 \mathrm{~cm}(4 \mathrm{MeV})$ and $0.7 \mathrm{~cm}$ $(15 \mathrm{MeV})$ for the $1 \times 1 \mathrm{~cm}^{2}$ field and decreased from $3.7 \mathrm{~cm}(4 \mathrm{MeV})$ to $1.6 \mathrm{~cm}(15 \mathrm{MeV})$ for $20 \times 20 \mathrm{~cm}^{2}$. Figure 3 (top) shows a plot of the relative penumbra on a semi-logarithmic scale expressed as a percentage of the corresponding field size. For the $4 \mathrm{MeV}$ case, the relative penumbra varies between $90 \%$ at $1 \times 1 \mathrm{~cm}^{2}$ 


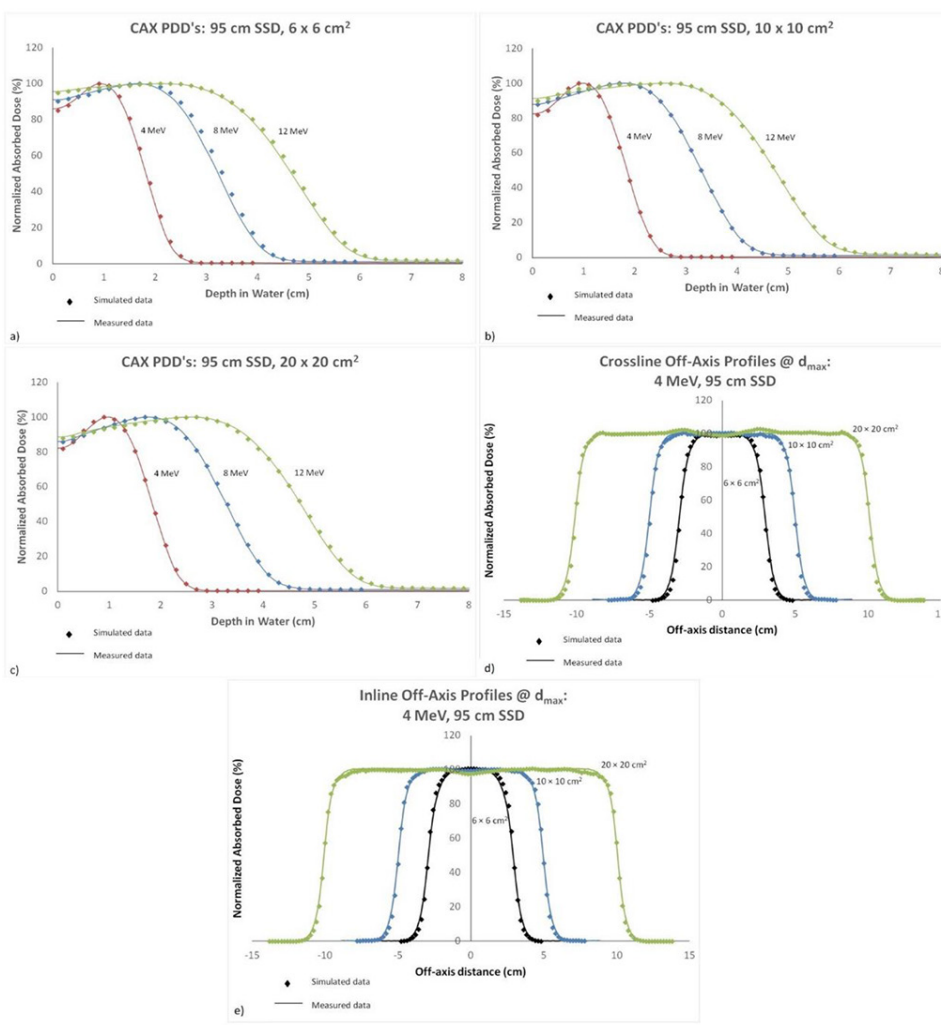

Figure 1: Monte Carlo (MC) (symbols) and measured (solid line) data comparison. In panels a) - c) corresponding central-axis (CAX) percentage depth dose (PDD) data is shown at $95 \mathrm{~cm}$ source-to-surface distance (SSD) for fields of $6 \times 6,10 \times 10$ and $20 \times 20 \mathrm{~cm}^{2}$, respectively. In panels d) and e), inline and crossline dose profile data at $4 \mathrm{MeV}$ are shown for the above three field sizes at 4, 8 and $12 \mathrm{MeV}$ measured at $95 \mathrm{~cm} \mathrm{SSD}$ at a depth of $0.9 \mathrm{~cm}$

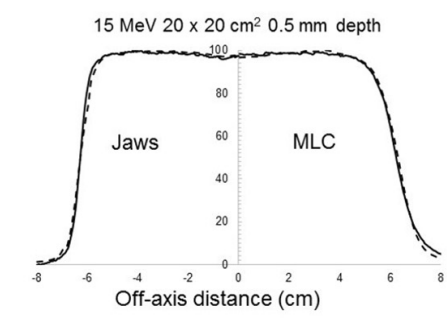

$4 \mathrm{MeV} 15 \times 15 \mathrm{~cm}^{2} 0.5 \mathrm{~mm}$ depth

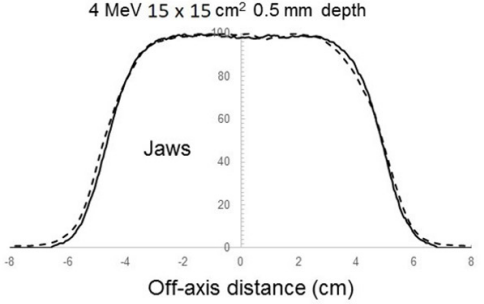

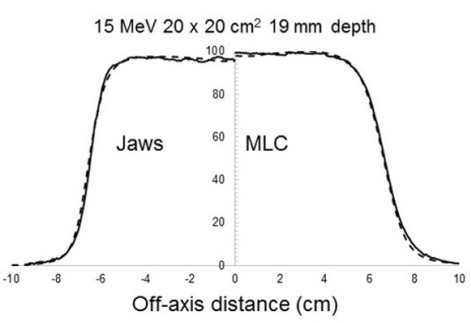

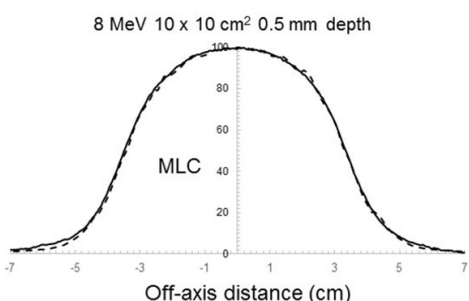

Figure 2: Comparison between Monte Carlo (MC) (broken lines) and film dose profiles (solid lines). In the top row half profiles are shown for the 'Jaws', and multi-leaf collimator ('MLC') collimated electron beams in a $20 \times 20 \mathrm{~cm}^{2}$ field at $15 \mathrm{MeV}$ for the top left: $0.5 \mathrm{~mm}$ depth, and top right: 19 $\mathrm{mm}$ depth. The row shows full profiles for the 'Jaws' (left: $4 \mathrm{MeV}, 15 \times 15 \mathrm{~cm}^{2}$ ) and the 'MLC' (right: $8 \mathrm{MeV}, 10 \times 10 \mathrm{~cm}^{2}$ ) fields. Field sizes were defined at $100 \mathrm{~cm}$ source-to-surface distance (SSD) 
MLC Segment Selection in Segmented-Field ECT

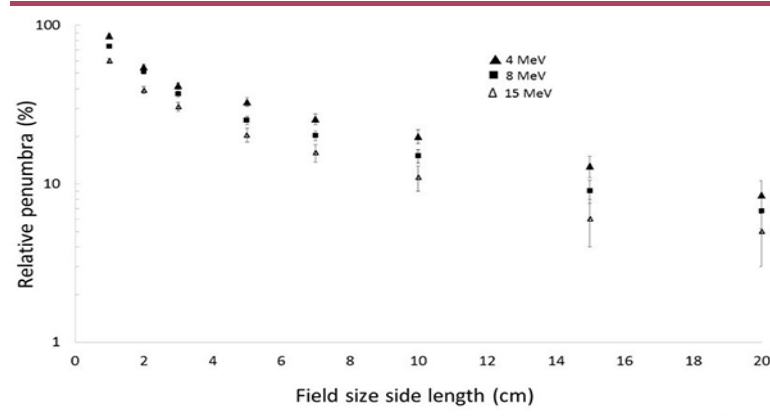

field and $9 \%$ at $20 \times 20 \mathrm{~cm}^{2}$. At $15 \mathrm{MeV}$, this variation is between $60 \%$ and $5 \%$ for $1 \times 1 \mathrm{~cm}^{2}$ and $20 \times 20 \mathrm{~cm}^{2}$ fields, respectively.

$\mathrm{R}_{90}$ depends on the field size and beam energy as shown in Figure 3 (middle). Superimposed on the data points are least-square fits of quadratic polynomials.

$\mathrm{R}_{90}$ was parameterised with a quadratic of the form:

$$
R_{90}(E)=a E^{2}+b E+c
$$

Different fitting parameters were used for each field size. Here, E represents the electron beam energy $(\mathrm{MeV})$. Calculated $\mathrm{R}_{90}$ values are within $0.1 \mathrm{~cm}$ of the values, shown in Figure 3 (middle).

\section{Percentage depth dose (PDD)}

Figure 3 (bottom) shows the sets of depth dose data at the indicated beam energies for a range of square MLC which collimated field sizes.

Bremsstrahlung dose (BSD) and dose fall-off range (DFR)

BSD enhancement makes a relatively larger contribution to the electron PDD beyond $\mathrm{R}_{\mathrm{p}}$ at low beam energies and small field sizes. At $4 \mathrm{MeV}$, the BSD contribution increases up to $11.3 \%$ for the $1 \times 1 \mathrm{~cm}^{2}$ field and declines due to the increase in the field size, which also includes data at higher energies.

In Table 1, a set of guidelines were defined for single field electron beams and the energy

Table 1: Guidelines were defined for single field electron beams

Field size $\left(\mathrm{cm}^{2}\right)$

\begin{tabular}{|c|c|c|c|c|c|c|c|c|}
\hline & $1 \times 1$ & $2 \times 2$ & $3 \times 3$ & $5 \times 5$ & $7 \times 7$ & $10 \times 10$ & $15 \times 15$ & $20 \times 20$ \\
\hline \multicolumn{9}{|c|}{ Energy range $\left(\mathrm{E}_{\text {lower }} / \mathrm{E}_{\text {upper }}\right)$} \\
\hline DFR $\leq 1.5$ & $4 / 6$ & $4 / 8$ & $4 / 8$ & $4 / 10$ & $4 / 10$ & $4 / 12$ & $4 / 12$ & $4 / 12$ \\
\hline BSD $\leq 5 \%$ & $8 / 15$ & $4 / 15$ & $4 / 15$ & $4 / 15$ & $4 / 15$ & $4 / 15$ & $4 / 15$ & $4 / 15$ \\
\hline$R_{90} / D F R \geq 1$ & - & $6 / 8$ & $6 / 10$ & $4 / 15$ & $4 / 15$ & $4 / 15$ & $4 / 15$ & $4 / 15$ \\
\hline
\end{tabular}

DFR: Dose fall-off range, BSD: Bremsstrahlung dose 
range. The guidelines of $\mathrm{DFR} \leq 1.5 \mathrm{~cm}$ were chosen to avoid excessive dose delivery to distal healthy tissue. For energies between 4 and $6 \mathrm{MeV}$, the $1 \times 1 \mathrm{~cm}^{2}$ field would fall within these guidelines. For the $7 \times 7 \mathrm{~cm}^{2}$ field, the energy range increases from 4 and $10 \mathrm{MeV}$ over which the DFR $\leq 1.5 \mathrm{~cm}$.

\section{Discussion}

Applicators with irregular cut-outs should not be used when using MLC collimated electron beams. It is necessary to study the properties of MLC collimated electron beams as far as their use in clinical cases. The first step was to build an accurate Monte Carlo model of the Elekta Precise linac. Results indicated that the Monte Carlo linac model could replicate depth dose and beam profiles delivered through standard applicators within $2 \% / 2$ $\mathrm{mm}$. As a further benchmark, Gafchromic film measurements indicated correspondence within $2 \% / 2 \mathrm{~mm}$ for MLC collimated and Monte Carlo simulated electron beams. From these measurements, the Monte Carlo model was deemed accurate. Dosimetric parameters were extracted from the PDD data (Figure 3, bottom) to develop single field selection guidelines as discussed below.

Larger fields have broader $\mathrm{P}_{80-20}$, (Figure 3, top), indicating more lateral dose involvement. An appropriate treatment margin should cover the tumour within the $90 \%$ dose level. In Figure 3 top, the relative penumbra width is nearly $100 \%$ for a $1 \times 1 \mathrm{~cm}^{2}$ field, reducing to a few percent for the $20 \times 20 \mathrm{~cm}^{2}$ field. For the smallest field size studied, the penumbra is almost as wide as this field.

$\mathrm{R}_{90}$ increases with increasing field size up to a critical field size (CFS) after which it remains invariant due to full lateral electron build-up (LEB). For $\mathrm{R}_{90}$ at a constant energy, it is observed that, at $4 \mathrm{MeV}$, this quantity does not change for field sizes larger than $2 \times 2 \mathrm{~cm}^{2}$ and for $6 \mathrm{MeV}$, the $\mathrm{CFS} \approx 3 \times 3 \mathrm{~cm}^{2}$ and at 8
$\mathrm{MeV}$ the $\mathrm{CFS}=5 \times 5 \mathrm{~cm}^{2}$. For $10 \mathrm{MeV}$, the $\mathrm{CFS} \approx 7 \times 7 \mathrm{~cm}^{2}$ and for beam energies $\geq 10$ $\mathrm{MeV}$, the $\mathrm{CFS}=10 \times 10 \mathrm{~cm}^{2}$.

The BSD depends on field size as well as beam energy. At $4 \mathrm{MeV}$, the BSD decreases rapidly from $11.3 \%$ for a $1 \times 1 \mathrm{~cm}^{2}$ field to $2.4 \%$ for a $2 \times 2 \mathrm{~cm}^{2}$ field due to LEB, increasing the electron dose contribution on the beam central axis. At $4 \mathrm{MeV}$, the BSD for a field size of $5 \times 5 \mathrm{~cm}^{2}$ has reached a constant value with increasing field size. As a rule, it can be stated that electron fields larger than the CFS have constant BSD contributions at a given beam energy.

The DFR reach constant values for fields which are more than CFS at the beam energy under consideration. Here, the central axis dose is fully enhanced by off-axis electrons, scattering into this region and resulting in shorter DFR values. Narrow beams (small fields) become increasingly more diffuse as the depth increases leading to longer DFR. It increases as a function of beam energy, regardless of the field size. Larger distal tumour depth would involve more healthy tissue. For smaller distal tumour depth, lower beam energy would be useful, resulting in a lesser dose to distal tissue due to lower DFR.

Larger electron fields have a wider energy range of application compared to smaller fields when were benchmarked against the guidelines. For example, if it is determined that a $5 \times 5 \mathrm{~cm}^{2}$ field is adequate for tumour coverage, it will meet all dosimetric guidelines set in Table 1 for beam energies between 4 and 10 $\mathrm{MeV}$. This is for cases where too much beam involvement into distal OAR's would present a challenge.

\section{Conclusion}

MC simulations agreed within $2 \% / 2 \mathrm{~mm}$ for PDD and beam profiles as benchmarked against water tank and Gafchromic film measurements. Analysis of the 3-D dose data 
MLC Segment Selection in Segmented-Field ECT

showed that fields smaller than $2 \times 2 \mathrm{~cm}^{2}$ should be avoided because of their higher BSD values and relative wide penumbras. Fields with sizes between $3 \times 3 \mathrm{~cm}^{2}$ and $10 \times$ $10 \mathrm{~cm}^{2}$ are preferred as they met the guidelines over the total range of electron beam energies in this study.

\section{Acknowledgment}

The authors thank Mrs. T. Mulder, medical editor, School of Medicine, University of the Free State, for technical and editorial preparation of the manuscript.

\section{Authors' Contribution}

FCP Du Plessis was involved in the writing, development of the methodology, and was responsible for the funding acquisition and supervision. D Van Eeden contributed to the writing, reviewing, and editing of the overall research and results. KN Sachse performed simulations and analyzed the results. All the authors read, modified, and approved the final version of the manuscript.

\section{Funding}

This research project was funded by the South African Medical Research Council in terms of the MRC's Flagships Awards Project [SAMRC-RFAUFSP-01-2013/HARD].

\section{Conflict of Interest}

None

\section{References}

1. Hogstrom KR, Boyd RA, Antolak JA, Svatos MM, Faddegon BA, Rosenman JG. Dosimetry of a prototype retractable eMLC for fixed-beam electron therapy. Med Phys. 2004;31(3):443-62. doi: 10.1118/1.1644516. PubMed PMID: 15070241.

2. Moran JM, Martel MK, Bruinvis IA, Fraass BA. Characteristics of scattered electron beams shaped with a multileaf collimator. Med Phys. 1997;24(9):1491-8. doi: 10.1118/1.598046. PubMed PMID: 9304578.

3. Xiong W, Li J, Chen L, Price RA, Freedman G, Ding M, Qin L, Yang J, Ma CM. Optimi- zation of combined electron and photon beams for breast cancer. Phys Med Biol. 2004;49(10):1973-89. doi: 10.1088/00319155/49/10/010. PubMed PMID: 15214536.

4. Hyödynmaa S, Gustafsson A, Brahme A. Optimization of conformal electron beam therapy using energy- and fluence-modulated beams. Med Phys. 1996;23(5):659-66. doi: 10.1118/1.597710. PubMed PMID: 8724738.

5. International Commission on Radiation Units and Measurements. Radiation Dosimetry: Electron beams with energies between 1 and $50 \mathrm{MeV}$ ICRU. Report ICRU 35; USA: ICRU Publ; 1984.

6. Olofsson L, Mu X, Nill S, Oelfke U, Zackrisson $B$, Karlsson $M$. Intensity modulated radiation therapy with electrons using algorithm based energy/range selection methods. Radiother Oncol. 2004;73(2):223-31. doi: 10.1016/j.radonc.2004.08.020. PubMed PMID: 15542170.

7. Mihaljevic J, Soukup M, Dohm 0, Alber M. Monte Carlo simulation of small electron fields collimated by the integrated photon MLC. Phys Med Biol. 2011;56(3):829-43. doi: $10.1088 / 0031-9155 / 56 / 3 / 018$. PubMed PMID: 21242628.

8. Henzen D, Manser P, Frei D, Volken W, Neuenschwander H, Born EJ, Vetterli D, Chatelain C, Stampanoni MF, Fix MK. Monte Carlo based beam model using a photon MLC for modulated electron radiotherapy. Med Phys. 2014;41(2):021714. doi: 10.1118/1.4861711. PubMed PMID: 24506605.

9. Salguero FJ, Arráns R, Palma BA, Leal A. Intensity- and energy-modulated electron radiotherapy by means of an XMLC for head and neck shallow tumors. Phys Med Biol. 2010;55(5):1413-27. doi: 10.1088/00319155/55/5/010. PubMed PMID: 20150682.

10. Hogstrom KR, Almond PR. Review of electron beam therapy physics. Phys Med Biol. 2006;51(13):R455-89. doi: 10.1088/00319155/51/13/R25. PubMed PMID: 16790918.

11.Du Plessis FC, Leal A, Stathakis S, Xiong W, Ma CM. Characterization of megavoltage 
electron beams delivered through a photon multi-leaf collimator (pMLC). Phys Med Biol. 2006;51(8):2113-29. doi: 10.1088/00319155/51/8/011. PubMed PMID: 16585849.

12.Kawrakow I. Accurate condensed history Monte Carlo simulation of electron transport. I. EGSnrc, the new EGS4 version. Med Phys. 2000;27(3):485-98. doi: 10.1118/1.598917. PubMed PMID: 10757601.

13.Rogers DW, Faddegon BA, Ding GX, Ma
CM, We J, Mackie TR. BEAM: a Monte Carlo code to simulate radiotherapy treatment units. Med Phys. 1995;22(5):503-24. doi: 10.1118/1.597552. PubMed PMID: 7643786.

14.0'Reilly D, Smit CJ, Du Plessis FC. Extraction of electron beam dose parameters from EBT2 film data scored in a mini phantom. Australas Phys Eng Sci Med. 2013;36(3):339-46. doi: 10.1007/s13246-013-0205-1. PubMed PMID: 23794059. 